\title{
Morphological stability of worm-like vesicles consisting of amphiphilic diblock copolymer against external stress
}

\author{
Eri Yoshida
}

\begin{abstract}
The morphological stability of vesicles consisting of an amphiphilic poly(methacrylic acid)block-poly(methyl methacrylate-random-methacrylic acid) diblock copolymer, PMAA- $b$-P(MMA-r-MAA), was investigated against the external stresses of $\mathrm{pH}$, salt concentration and polyamine. The worm-like vesicles underwent a partial fusion at $\mathrm{pH} \mathrm{12,} \mathrm{however,} \mathrm{they} \mathrm{retained} \mathrm{the} \mathrm{worm-like} \mathrm{shape} \mathrm{at} \mathrm{pH} 13$ due to electrostatic

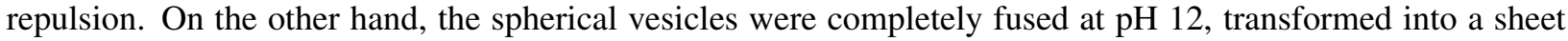
and did not retain their shape under the higher basic condition. Similarly, the worm-like vesicles retained their morphology in $0.1 \mathrm{~mol} \%$ solutions of sodium chloride and sodium dodecyl sulfate, while the spherical vesicles caused division and fusion even at much lower concentrations. Poly(2-dimethylaminoethyl methacrylate) (PDMAEMA) transformed the worm-like vesicle into a cleavable sheet, while it changed the spherical vesicles into a sheet without a specific form. It was found that this transformation based on the acid-base interaction between the carboxylic acid of the MAA block and the amine of the PDMAEMA was dependent on the molecular weight of the PDMAEMA. The short PDMAEMA retarded the fusion of the vesicles.
\end{abstract}

Keywords: morphological stability, external stresses, worm-like vesicles, spherical vesicles, amphiphilic diblock copolymer, polyamine

\section{Introduction}

Single-cell bacteria have many different morphologies. They include spherical Staphylococcus aureus, ${ }^{[1,2]}$ rod-like Bacillus subtilis, ${ }^{[3,4]}$ long worm-like Escherichia coli ${ }^{[2,4]}$ and Bifidobacteria ${ }^{[5]}$ and spiral Spirochete ${ }^{[6]}$ and Campylobacter pylori. ${ }^{[7]}$ Organelles in living cells also have various morphologies based on the specific compositions of the lipids with their individual critical packing shapes that determine the bilayer thickness, curvature, and shapes. ${ }^{[8,9]}$ Examples include the elliptical mitochondria, ${ }^{[10]}$ tubular microvilli of the epithelial cell, ${ }^{[11]}$ and the interconnected tubular network of the surfaces of the endoplasmic reticulum ${ }^{[12]}$ and Golgi apparatus. $^{[13]}$ The morphologies of the bacteria and organelles often change responding to the circumstances and external stresses. ${ }^{[14-16]}$

Vesicles composed of amphiphilic diblock copolymers are possible artificial and morphological mod-

Received: April 25, 2019 Accepted: May 27, 2019 Published: May 28, 2019

* Correspondence to: Eri Yoshida, Department of Applied Chemistry and Life Science, Toyohashi University of Technology, 1-1 Hibarigaoka, Tempaku-cho, Toyohashi, Aichi 441-8580, Japan; Email: eyoshida@ chem.tut.ac.jp

Citation: Yoshida E. Morphological stability of worm-like vesicles consisting of amphiphilic diblock copolymer against external stress. Chem Rep, 2019, 1(2): 102-107.

Copyright: (c) 2019 Eri Yoshida. This is an open access article distributed under the terms of the Creative Commons Attribution License, which permits unrestricted use, distribution, and reproduction in any medium, provided the original author and source are credited. els of biomembranes for the bacteria and organelles based on the similarities in their size and structure. ${ }^{[17]}$ Some morphologies have already been created using poly(methacrylic acid)-block-poly(methyl methacrylaterandom-methacrylic acid), PMAA- $b$-P(MMA-r-MAA) by polymerization-induced self-assembly using the nitroxide-mediated photo controlled/living radical polymerization technique (photo NMP). ${ }^{[18]}$ The morphologies include spherical vesicles, ${ }^{[17]}$ the elliptical, ${ }^{[19]}$ worm-like, ${ }^{[20,21]}$ key-shaped, ${ }^{[22]}$ villus-like, ${ }^{[23]}$ and anastomosed tubular networks. ${ }^{[24]}$ These morphologies were determined by the block length and molar ratio of the monomer unit in the diblock copolymer. The copolymer also provided new models relating to the membrane permeability, ${ }^{[25]}$ budding separation during endocytosis, ${ }^{[26]}$ and sterol intercalation. ${ }^{[27]}$ It is important to investigate the morphological stability of the vesicles against the external stress in order to elucidate the biomembranes of the bacteria and organelles from a morphological aspect. The difference in thermal stability has already been reported between the worm-like vesicles and spherical vesicles consisting of PMAA- $b$-P(MMA$r$-MAA). ${ }^{[2]}$ This paper describes the morphological stability of these vesicles against $\mathrm{pH}$, salt concentration, and a basic polymer. 


\section{Experimental}

\subsection{Instrumentation}

Field emission scanning electron microscopy (FESEM) measurements were performed using a Hitachi SU8000 scanning electron microscope.

\subsection{Materials}

The worm-like vesicles and spherical vesicles were prepared as reported previously. ${ }^{[22]} \operatorname{Poly}(2-$ dimethylaminoethyl methacrylate) (PDMAEMA) was prepared by the photo NMP. ${ }^{[28]}$ Methanol $(\mathrm{MeOH})$ was refluxed over magnesium with a small amount of iodine for several hours, and then distilled. Distilled water was purchased from Wako Pure Chemical Industries and used without further purification. Extra pure sodium chloride and sodium dodecyl sulfate (SDS) were purchased from Wako Pure Chemical and used without further purification.

\subsection{Stability against pH}

The worm-like vesicles $(5.1 \mathrm{mg})$ were dispersed in a $\mathrm{NaOH}$ aqueous solution $(4 \mathrm{~mL})$ with different $\mathrm{pH}$ by vigorous shaking. The dispersion was stood at room temperature for a week. The precipitates were isolated by decantation and dried in air for several hours. The product was subjected to the FE-SEM observation.

\subsection{Stability against salt concentration}

The vesicles $(5.6 \mathrm{mg})$ were dispersed in an aqueous $\mathrm{MeOH}$ solution $(0.8 \mathrm{~mL}, \mathrm{MeOH} /$ water $=3 / 1 \mathrm{v} / \mathrm{v})$ with different $\mathrm{NaCl}$ concentrations of $0.1,0.01$, and $10^{-3}$ mol\% by vigorous shaking. The mixture was stood at room temperature for 2 weeks. The precipitates were isolated by decantation and dried in air for several hours. The white powder product was subjected to the FE-SEM observation.

\subsection{Stability against PDMAEMA}

The vesicles $(5.1 \mathrm{mg})$ were placed in an aqueous $\mathrm{MeOH}$ solution $(3 \mathrm{~mL}, \mathrm{MeOH} / \mathrm{water}=3 / 1 \mathrm{v} / \mathrm{v})$. The PDMAEMA with $\mathrm{Mn}=40,100(30 \mathrm{mg})$ was dissolved in an aqueous $\mathrm{MeOH}$ solution ( $3 \mathrm{~mL}$ ). The PDMAEMA solution $(0.25 \mathrm{~mL})$ and an aqueous $\mathrm{MeOH}$ solution $(0.75$ $\mathrm{mL}$ ) were added to the vesicle solution. The mixture was dispersed by vigorous shaking and stood at room temperature for 2 weeks. The precipitates were isolated by decantation and dried in air for several hours. The product was subjected to the FE-SEM observation.

\subsection{FE-SEM observations}

The morphologies of the vesicles were observed using FE-SEM at $1.0 \mathrm{kV}$ without any coating of the vesicles.

\section{Results and Discussion}

The morphological stability against $\mathrm{pH}$ was investigated using the worm-like vesicles consisting of $\mathrm{PMAA}_{351}-b-\mathrm{P}\left(\mathrm{MMA}_{0.826}-r-\mathrm{MAA}_{0.174}\right)_{372}$. Figure 1 shows the variation in the morphology of the worm-like vesicles placed in different $\mathrm{pH}$ solutions. The vesicles retained the worm-like shape up to $\mathrm{pH} 11$ and partially fused at $\mathrm{pH} 12$. However, they retained their shape at $\mathrm{pH}$ 13. It is considered that at $\mathrm{pH} 12$, the partial ionization of the PMAA block caused the fusion of the vesicles by the hydrogen bonding interaction between an ionized carboxylate anion and a non-ionized carboxylic acid on the surface of the vesicle shells (Figure 2). At pH 13, the electrostatic repulsion between the carboxylate anions retained the worm-like morphology based on completed ionization. Many worm-like vesicles attached to a concave spherical vesicle were observed at $\mathrm{pH} 13$. The concave spherical vesicles were probably formed by partial swelling of a worm-like vesicle due to the intravesicularly electrostatic repulsion followed by its collapse. The $\mathrm{pH}$-responsiveness of the spherical vesicles based on a light scattering study has already been reported. ${ }^{[29]}$ However, the study did not include the morphological observations for each $\mathrm{pH}$. The spherical vesicles consisting of $\mathrm{PMAA}_{227}-b-\mathrm{P}\left(\mathrm{MMA}_{0.841}-r-\mathrm{MAA}_{0.159}\right)_{321}$ retained their shape up to $\mathrm{pH} 11$ as did the worm-like vesicles. The spherical vesicles were completely fused into a sheet-like morphology at pH 12 (Figure 3), however, they provided a nonspecific morphology at $\mathrm{pH} 13$ due to partial phase separation based on the electrostatic repulsion. For the spherical vesicles, the partial change affected the entire morphological change which caused the irreparable disruption.
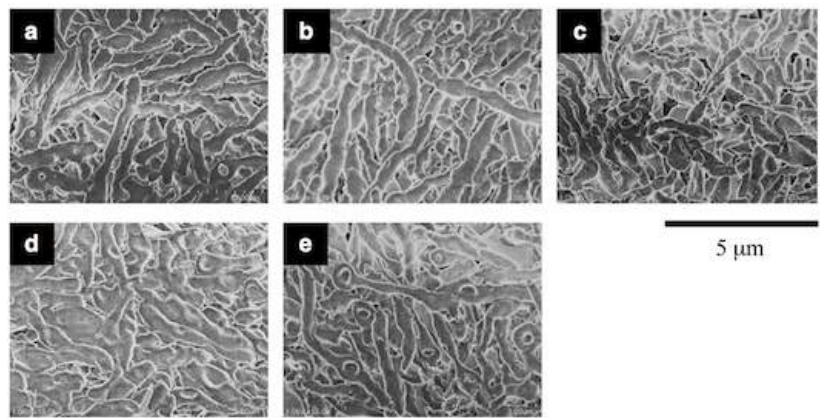

$5 \mu \mathrm{m}$

Figure 1. FE-SEM images of the worm-like vesicles at (a) $\mathrm{pH}$ 7 , (b) 9, (c) 11 , (d) 12 , and (e) 13 . [vesicle] $=1.28 \mathrm{~g} / \mathrm{L}$ 
a

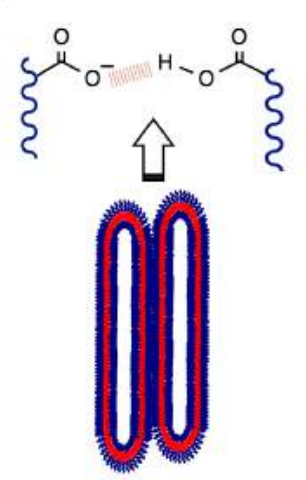

b

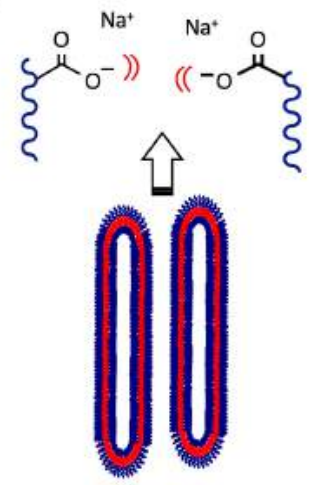

Figure 2. Schemes for (a) the fusion of the vesicles by the hydrogen bonding interaction between an ionized carboxylate anion and a non-ionized carboxylic acid and (b) the retention of the worm-like shape by the electrostatic repulsion between the carboxylate anions
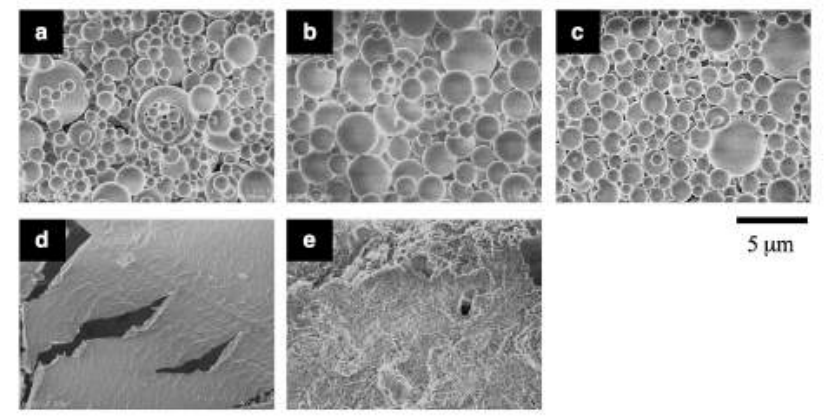

Figure 3. FE-SEM images of the spherical vesicles at (a) $\mathrm{pH} 7$, (b) 9, (c) 11 , (d) 12 , and (e) 13 . [vesicle] $=1.28 \mathrm{~g} / \mathrm{L}$

The stability of the vesicles against the salt concentration was also examined using $\mathrm{NaCl}$. The worm-like vesicles and spherical vesicles retained their shapes at $10^{-3} \mathrm{~mol} \%$ (Figure 4). The worm-like vesicles retained the morphology even at $0.1 \mathrm{~mol} \%$, whereas the spherical vesicles combined into larger vesicles at $0.01 \mathrm{~mol} \%$. The large vesicles were further fused into a sheet-like morphology at $0.1 \mathrm{~mol} \%$ based on the interaction between the PMAA blocks via $\mathrm{Na}^{+}$(Figure 5). SDS also caused morphological transformation of the vesicles. However, the salt produced a change opposite to that of the spherical vesicles by $\mathrm{NaCl}$. SDS partially swelled the wormlike vesicles without fusion even at $0.1 \mathrm{~mol} \%$ (Figure 6), whereas the salt divided the spherical vesicles into much smaller spheres at $0.01 \mathrm{~mol} \%$. SDS with a cone-like critical packing shape due to the long hydrophobic hydrocarbon chain was intercalated into the hydrophobic cores of the vesicles causing an increase in the curvature of the bilayer to produce much smaller vesicles. These small vesicles were further fused into a sheet at $0.1 \mathrm{~mol} \%$.

Polyamine has the potential to also serve as an ex-
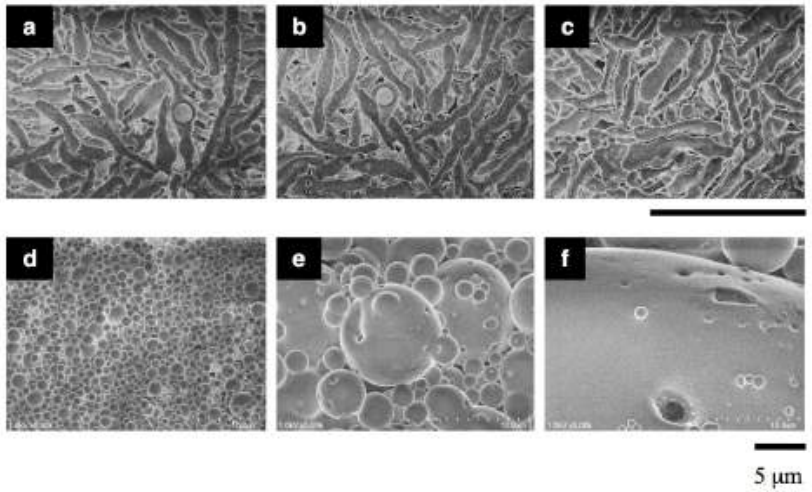

Figure 4. FE-SEM images of the worm-like vesicles in a $\mathrm{NaCl}$ solution at (a) $10^{-3}$, (b) 0.01 , and (c) $0.1 \mathrm{~mol} \%$ and the spherical vesicles at (d) $10^{-3}$, (e) 0.01 , and (f) $0.1 \mathrm{~mol} \%$. [vesicle] $=7.00$ $\mathrm{g} / \mathrm{L}$. Bars $=5 \mu \mathrm{m}$

ternal stress for the vesicles as well as $\mathrm{NaCl}$ and SDS based on the acid-base interaction on the vesicle shells. The morphological stability of the vesicles was evaluated using PDMAEMA with $\mathrm{Mn}=40,100(\mathrm{Mw} / \mathrm{Mn}=$ 1.89). When the worm-like vesicles were placed in a solution containing PDMAEMA at 0.1 as a molar ratio of the amino group (Am) to the carboxylic acid (Cb), part of the vesicle transformed into a cup-like shape to produce a scoop-like vesicle (Figure 7). It has been reported that the cup-like morphology was formed in the early stage of the polymerization during the process of preparing the worm-like vesicles. ${ }^{[20]}$ The copolymer with the short hydrophobic chain during the early stage was considered to form the rim of the cup-like vesicle based on its cone-like critical packing shape. The acid-base interaction between the amine and carboxylic acid increases the hydrophilic surface area of the critical packing shape of the copolymer due to the more hydrophilic salt formation causing a change in the critical packing shape into a cone-like shape. An increase in the PDMAEMA concentration to $\mathrm{Am} / \mathrm{Cb}=0.25$ transformed the vesicles into short worm-like or small spherical vesicles. A further increase to $\mathrm{Am} / \mathrm{Cb}=0.5$ changed them into interconnected vesicles, followed by a partial sheet at $\mathrm{Am} / \mathrm{Cb}=1.0$, and finally into a cleavable sheet at $\mathrm{Am} / \mathrm{Cb}=2.0$. PDMAEMA also produced morphological changes in the spherical vesicles (Figure 8). The vesicles were disrupted into much smaller particles along with a small amount of cup-like vesicles at $\mathrm{Am} / \mathrm{Cb}$ $=0.1$. At $\mathrm{Am} / \mathrm{Cb}=0.25$, partial fusion of the vesicles occurred, indicating that the spherical vesicles were more easily deformed than the worm-like vesicles by the acid-base interaction. As the amine concentration increased to $\mathrm{Am} / \mathrm{Cb}=0.5$, the vesicles transformed into a partial sheet, followed by a flexible sheet at $\mathrm{Am} / \mathrm{Cb}$ 


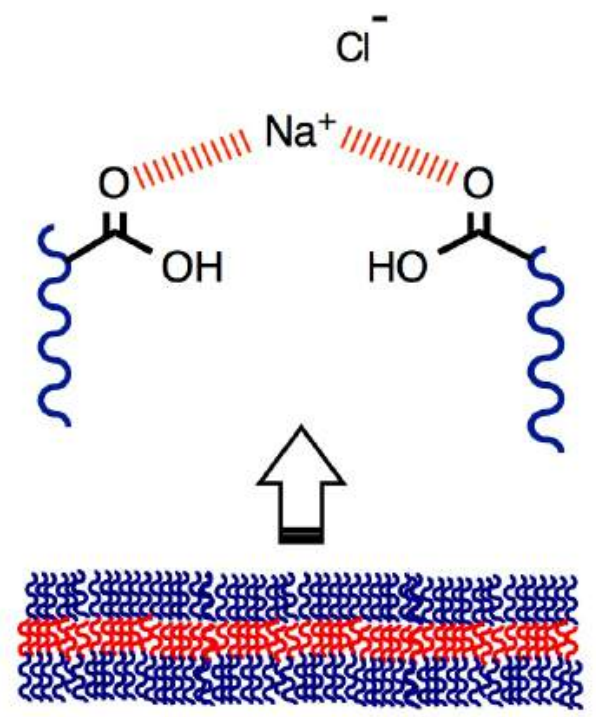

Figure 5. A scheme for the interaction between the PMAA blocks via $\mathrm{Na}^{+}$in a sheet-like morphology

$=1.0$, and finally into a completed sheet at $\mathrm{Am} / \mathrm{Cb}=$ 2.0. The morphological change in the vesicles was dependent on the molecular weight of the polyamine. As can be seen in Figure 9, the PDMAEMA with a lower molecular weight of $\mathrm{Mn}=13,100(\mathrm{Mw} / \mathrm{Mn}=1.56) \mathrm{re}$ tarded the fusion of the worm-like vesicles at the same amine concentration. The morphology at $\mathrm{Am} / \mathrm{Cb}=0.5$ for the short PDMAEMA was equal to that at $\mathrm{Am} / \mathrm{Cb}$ $=0.1$ for the long PDMAEMA. The spherical vesicles were fused even by the short PDMAEMA, however, the fusion produced a sheet with a much smaller area by the short PDMAEMA than the long one. The retardation of the fusion by the short PDMAEMA is due to the fact that the short PDMAEMA forms the intravesicular
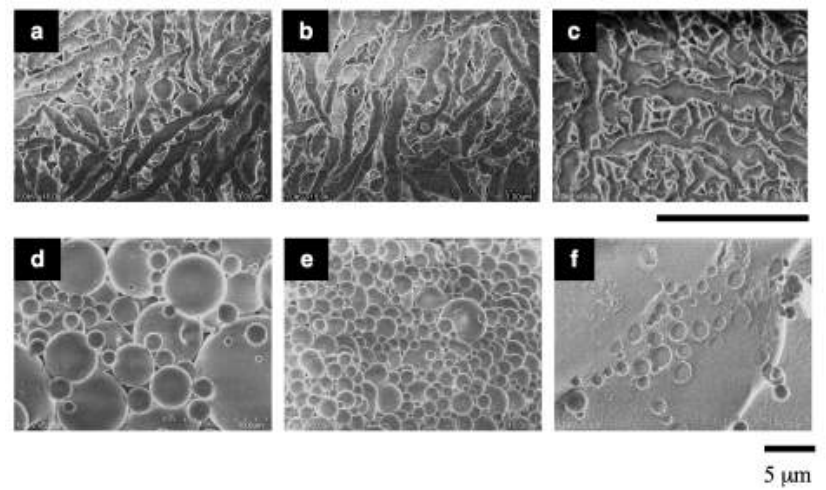

Figure 6. FE-SEM images of the worm-like vesicles in an SDS solution at (a) $10^{-3}$, (b) 0.01 , and (c) $0.1 \mathrm{~mol} \%$ and the spherical vesicles at (d) $10^{-3}$, (e) 0.01 , and (f) $0.1 \mathrm{~mol} \%$. [vesicle] $=7.00$ $\mathrm{g} / \mathrm{L}$. Bars $=5 \mu \mathrm{m}$
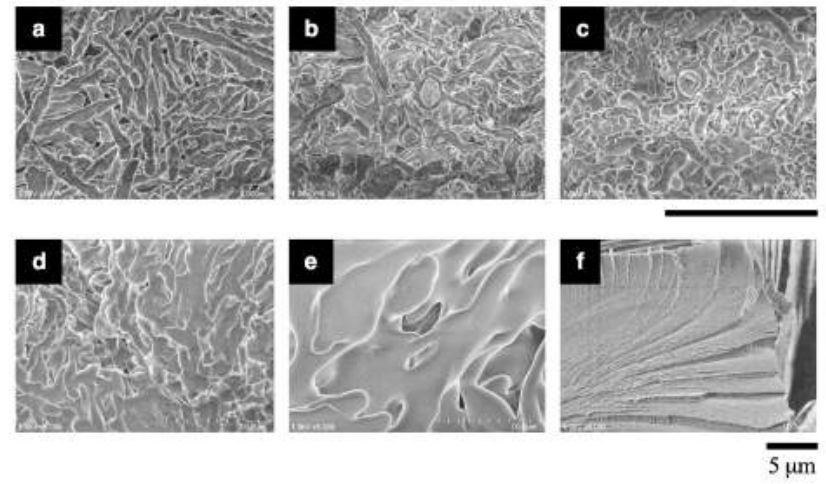

Figure 7. FE-SEM images of the worm-like vesicles in a PDMAEMA solution at $\mathrm{Am} / \mathrm{Cb}=$ (a) 0 , (b) 0.1 , (c) 0.25 , (d) 0.5 , (e) 1.0, and (f) 2.0. PDMAEMA: $\mathrm{Mn}=40,100$. [vesicle] $=1.28$ $\mathrm{g} / \mathrm{L}$. Bars $=5 \mu \mathrm{m}$
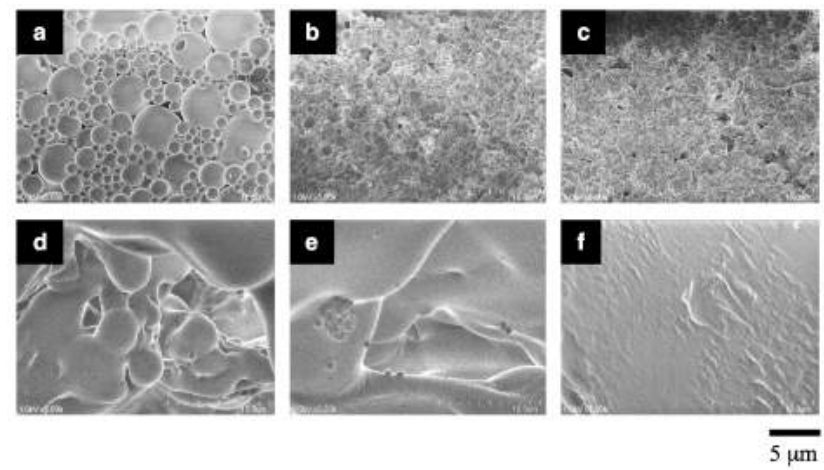

Figure 8. FE-SEM images of the spherical vesicles consisting of PMAA $214-b$-P(MMA M. $_{037}-r$-MAA M. $\left._{0.163}\right)_{348}$ in a PDMAEMA solution at $\mathrm{Am} / \mathrm{Cb}=$ (a) 0, (b) 0.1, (c) 0.25, (d) 0.5, (e) 1.0, and (f) 2.0. PDMAEMA: $\mathrm{Mn}=40,100$. [vesicle] $=1.28 \mathrm{~g} / \mathrm{L}$

cross-linkage between the PMAA block chains based on the acid-base interaction (Figure 10), whereas the long PDMAEMA causes the intervesicular cross-linking.

\section{Conclusion}

This is the first study that demonstrated the difference in the morphological stability between the wormlike vesicles and spherical vesicles against the external stresses. The worm-like vesicles were fused under a basic condition, however, they retained their worm-like shape at a much higher basicity due to the electrostatic repulsion. On the other hand, the spherical vesicles were easily fused into a sheet and did not retain their shape at the highly basicity. Similarly, the worm-like vesicles retained their morphology even at the high concentrations of $\mathrm{NaCl}$ and SDS, while the spherical vesicles caused division and fusion at much lower concentrations. The acid-base interaction with the polyamine also produced changes in the vesicles depending on its molecular weight. The short polyamine retarded the fusion of 

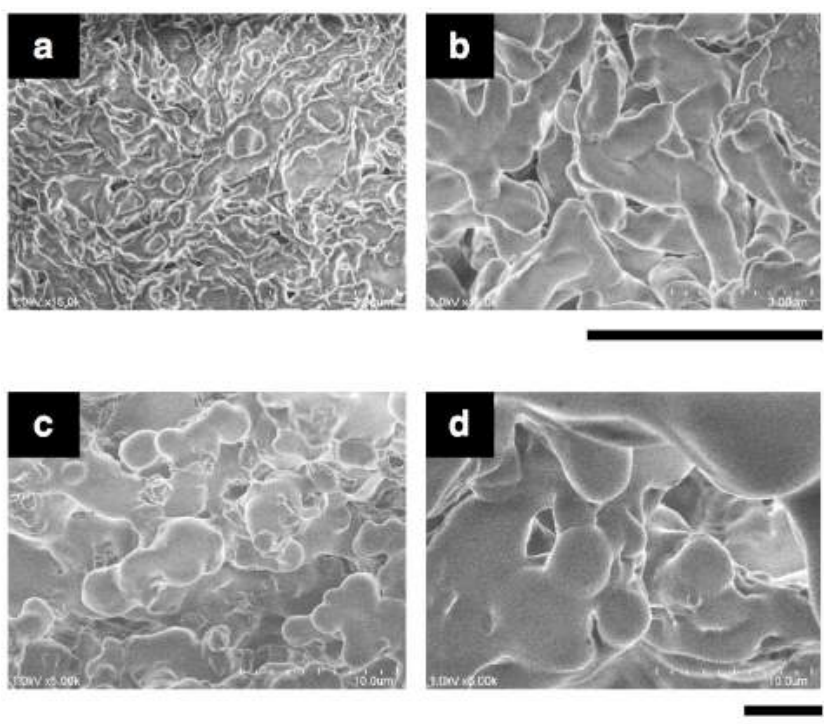

$5 \mu \mathrm{m}$

Figure 9. FE-SEM images of the worm-like vesicles (a and $b$ ) and the spherical vesicles consisting of PMAA 214- $^{-}$ $b$-P(MMA $\left.{ }_{0.837}-r-\mathrm{MAA}_{0.163}\right)_{348}(\mathrm{c}$ and $\mathrm{d})$ in a solution of PDMAEMA with $\mathrm{Mn}=(\mathrm{a}$ and c) 13,100 and $(\mathrm{b}$ and d) 40,100 $\mathrm{Am} / \mathrm{Cb}=0.5$. [worm-like vesicle] $=1.70 \mathrm{~g} / \mathrm{L}$, [spherical vesicle] $=1.73 \mathrm{~g} / \mathrm{L}$. Bars $=5 \mu \mathrm{m}$

a

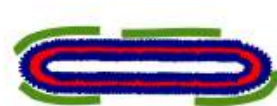

b

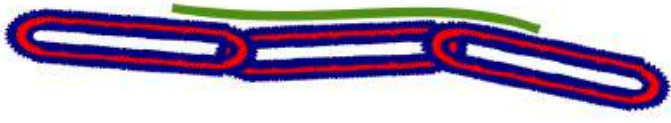

PDMAEMA

Figure 10. Schemes for (a) the intravesicular cross-linking by the short PDMAEMA and (b) intervesicular cross-linking by the long one

the vesicles. These findings are useful for manipulating the controlled release of substances encapsulated in the vesicles.

\section{Conflict of Interest}

The author has no conflict of interest in this study.

\section{Acknowledgment}

The author is thankful for a JSPS Grant-in-Aid for Scientific Research (Grant Number 18K04863).

\section{References}

[1] Anderson RC, Haverkamp RG and Yu PL. Investigation of morphological changes to Staphylococcus aureus induced by ovine-derived antimicrobial peptides using TEM and AFM. FEMS Microbiology Letters, 2004, 240: 105-110. https://doi.org/10.1016/j.femsle.2004.09.027

[2] Braga PC, Sasso MD and Maci S. Cefodizime: effects of sub-inhibitory concentrations on adhesiveness and bacterial morphology of Staphylococcus aureus and Escherichia coli: comparison with cefotaxime and ceftriaxone. Journal of Antimicrobial Chemotherapy, 1997, 39(1): 79-84.

https://doi.org/10.1093/jac/39.1.79

[3] Reeve JN, Mendelson NH and Cole RM. Cell morphology of Bacillus subtilis: The effect of genetic background on the expression of a rod- gene. Molecular \& General Genetics Mgg, 1972, 119(1): 11-26. https://doi.org/10.1007/BF00270440

[4] Li H, Chen Q, Zhao J, et al. Enhancing the antimicrobial activity of natural extraction using the synthetic ultrasmall metal nanoparticles. Scientific Reports, 2015, 5: 11033. https://doi.org/10.1038/srep11033

[5] Scardovi V and Trovatelli LD. Bifidobacterium animalis (Mitsuoka) comb. nov. and the "minimum" and "subtile" groups of new bifidobacteria found in sewage. International Journal of Systematic Bacteriology, 1974, 24: 21-28. https://doi.org/10.1099/00207713-24-1-21

[6] Hampp EG, Scott DB and Wyckoff RWG. Morphologic characteristics of certain cultured strains of oral Spirochetes and Treponema pallidum as revealed by the electron microscope. Journal of Bacteriology, 1948, 56(6): 755-769. https://doi.org/10.1007/BF00446581

[7] McNulty CAM, Dent JC, Curry A, et al. New spiral bacterium in gastric mucosa. Journal of Clinical Pathology, 1989, 42(6): 585-591. https://doi.org/10.1136/jcp.42.6.585

[8] Israelachvili JN, Mitchell DJ, and Ninham BW. Theory of self-assembly of hydrocarbon amphiphiles into micelles and bilayers. Journal of the Chemical Society, Faraday Transactions 2: Molecular and Chemical Physics, 1976, 72: 15251568. https://doi.org/10.1039/F29767201525

[9] Israelachvili JN, Intermolecular and Surface Forces, 3rd edn, Academic Press, Waltham, 2011.

[10] Frey TG and Mannella CA. The internal structure of mitochondria. Trends in Biochemical Sciences, 2000, 25(7): 319-324. https://doi.org/10.1016/S0968-0004(00)01609-1

[11] Kessel RG and Kardon RH. Tissues and Organs: A TextAtlas of Scanning Electron Microscopy. W. H. Freeman and Company, San Francisco, 1979, p.199.

[12] Selman GG, and Jurand A. An electron microscope study of the endoplasmic reticulum in newt notochord cells after disturbance with ultrasonic treatment and subsequent regeneration. The Journal of Cell Biology, 1964, 20(1): 175-183. https://doi.org/10.1083/jcb.20.1.175

[13] Orci L, Ravazzola MS, Amherdt M, et al. Bidirectional transport by distinct populations of COPI-coated vesicles. Cell, 1997, 90: 335-349. https://doi.org/10.1016/S0092-8674(00)80341-4 
[14] Burdett IDJ. Electron microscope study of the rod-to-coccus shape change in a temperature-sensitive rod- mutant of Bacillus subtilis. Journal of Bacteriology, 1979, 137(3): 1395-1405.

[15] Pérez-Núñez D, Briandet R, David B, et al. A new morphogenesis pathway in bacteria: unbalanced activity of cell wall synthesis machineries leads to coccus-to-rod transition and filamentation in ovococci. Molecular Microbiology, 2011, 79(3): 759-771. https://doi.org/10.1111/j.1365-2958.2010.07483.x

[16] Sheetz MP, Painter RG and Singer SJ. Biological membranes as bilayer couples III. Compensatory shape changes induced in membranes. The Journal of Cell Biology, 1976, 70(1): 193-203. https://doi.org/10.1083/jcb.70.1.193

[17] Yoshida E. Giant vesicles prepared by nitroxide-mediated photo-controlled/living radical polymerization-induced self-assembly. Colloid and Polymer Science, 2013, 291(11): 2733-2739. https://doi.org/10.1007/s00396-013-3056-0

[18] Yoshida E. Nitroxide-mediated photo-controlled/living radical polymerization of methacrylic acid. Open Journal of Polymer Chemistry, 2013, 3:16-22. https://doi.org/10.4236/ojpchem.2013.31004

[19] Yoshida E. Morphology transformation of micrometersized giant vesicles based on physical conditions for photopolymerization-induced self-assembly. Supramolecular Chemistry, 2015, 27(4): 274-280. https://doi.org/10.1080/10610278.2014.959014

[20] Yoshida E. Giant vesicles comprised of mixed amphiphilic poly(methacrylic acid)-block-poly(methyl methacrylaterandom-methacrylic acid) diblock copolymers. Colloid and Polymer Science, 2016, 293: 3641-3648. https://doi.org/10.1007/s00396-015-3763-9

[21] Yoshida E. Worm-like vesicle formation by photocontrolled/living radical polymerization-induced selfassembly of amphiphilic poly(methacrylic acid)-blockpoly(methyl methacrylate-random-methacrylic acid). Colloid and Polymer Science, 2016, 294: 1857-1863. https://doi.org/10.1007/s00396-016-3935-2
[22] Yoshida E. Morphology control of giant vesicles by composition of mixed amphiphilic random block copolymers of poly(methacrylic acid)-block-poly(methyl methacrylaterandom-methacrylic acid). Colloid and Polymer Science, 2015, 293: 249-256.

https://doi.org/10.1007/s00396-014-3403-9

[23] Yoshida E. Fabrication of microvillus-like structure by photopolymerization-induced self-assembly of an amphiphilic random block copolymer. Colloid and Polymer Science, 2015, 293: 1841-1845. https://doi.org/10.1007/s00396-015-3600-1

[24] Yoshida E. Fabrication of anastomosed tubular networks developed out of fenestrated sheets through thermo responsiveness of polymer giant vesicles. ChemXpress, 2017, 10(1): 118

[25] Yoshida E. Enhanced permeability of Rhodamine B into bilayers comprised of amphiphilic random block copolymers by incorporation of ionic segments in the hydrophobic chains. Colloid and Polymer Science, 2015, 293: 2437 2443. https://doi.org/10.1007/s00396-015-3679-4

[26] Yoshida E. Morphology transformation of giant vesicles by a polyelectrolyte for an artificial model of a membrane protein for endocytosis. Colloid Surface Science, 2018, 3: 6-11. https://doi.org/10.11648/j.css.20180301.12

[27] Yoshida E. Morphological changes in polymer giant vesicles by intercalation of a segment copolymer as a sterol model in plasma membrane. Colloid and Polymer Science, 2015, 293: $1835-1840$. https://doi.org/10.1007/s00396-015-3577-9

[28] Yoshida E. Photo-controlled/living radical polymerization of 2-(dimethylamino)ethylmethacrylate using 4-methoxy2,2,6,6-tetramethylpiperidine-1-oxyl as a mediator. Colloid and Polymer Science, 2012, 290: 965-969. https://doi.org/10.1007/s00396-012-2641-y

[29] Yoshida E. PH response behavior of giant vesicles comprised of amphiphilic poly(methacrylic acid)-blockpoly(methyl methacrylate-random-methacrylic acid). Colloid and Polymer Science, 2015, 293: 649-653. https://doi.org/10.1007/s00396-014-3482-7 